\title{
Impact of chronic consumption of dairy products varying in fatty acid composition on postprandial lipid responses: preliminary insights from the RESET study
}

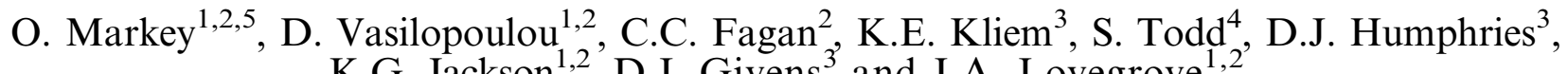 \\ K.G. Jackson ${ }^{1,2}$, D.I. Givens ${ }^{3}$ and J.A. Lovegrove ${ }^{1,2}$ \\ ${ }^{1}$ Hugh Sinclair Unit of Human Nutrition and Institute for Cardiovascular and Metabolic Research, ${ }^{2}$ Department of \\ Food and Nutritional Sciences, ${ }^{3}$ Institute for Food, Nutrition and Health, University of Reading, ${ }^{4}$ Department of \\ Mathematics and Statistics, University of Reading, Reading, RG6 6AP and ${ }^{5}$ School of Sport, Exercise and Health \\ Sciences, Loughborough University, Loughborough, LE11 3TU, UK.
}

Partial replacement of milk saturated fatty acids (SFA) with unsaturated fatty acids (FA) is feasible through supplementation of the bovine diet with plant oil or oilseeds ${ }^{(1)}$. Consumption of modified dairy products with a SFA-reduced, monounsaturated fat (MUFA)-enriched content may have a beneficial impact on the fasting lipid profile ${ }^{(1)}$ but their influence on postprandial triacylglycerol (TAG) concentrations, an independent risk factor for $\mathrm{CVD}^{(2)}$, requires investigation. We examined whether consumption of FA-modified dairy products improved postprandial lipid, glucose and insulin responses, when compared to dairy products with a FA composition typical of retail products (control).

We conducted a 12-week, randomised, crossover, double-blinded controlled dietary intervention in fifty-two adults at moderate CVD risk (31 men, 21 women; age 53 (SE 2) years; BMI $25.9($ SE 0.5) kg/m²) (REplacement of SaturatEd fat in dairy on Total cholesterol (RESET) study; ClinicalTrials.gov: NCT02089035). A flexible food-exchange model was used to implement each iso-energetic high-fat (38\%TE), high-dairy diet that contained UHT milk, cheese and butter: control (dietary target: $19 \% \mathrm{TE}$ SFA; $11 \% \mathrm{TE}$ MUFA) and modified (16\%TE SFA; $14 \%$ TE MUFA). Before and after each intervention period, participants underwent a sequential two-meal postprandial investigation in which blood samples were collected at regular intervals following a test breakfast (50 g fat) and lunch (30 g fat) rich in control or modified dairy products, given at 0 and 330 min respectively. Changes from the baseline study visit in postprandial serum TAG, glucose, apolipoprotein B (apoB) and non-esterified FAs (NEFA) and plasma insulin response were reported as incremental area under the curve (iAUC; Fig. 1.). Data were analysed using mixed models, with baseline values of the assessed variable, period, treatment, age, gender and BMI included as fixed effects.
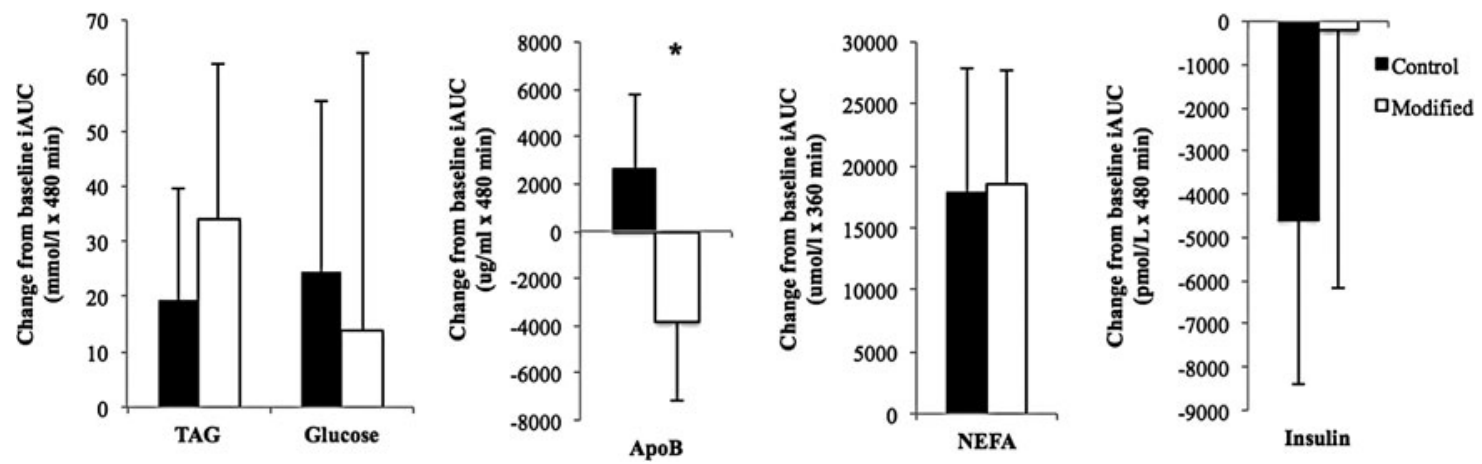

Fig. 1. Change from the baseline study visit in postprandial lipid, glucose and insulin responses following consumption of sequential high-fat meals that incorporated control and modified dairy products. Values are means with their standard errors represented by vertical bars. $* P<0 \cdot 01$ (treatment effect). iAUC, incremental area under the curve; TAG, triacylglycerol; apoB, apolipoprotein B; NEFA, non-esterified FAs.

A differential effect was observed for the apoB iAUC, with a decrease observed following the modified diet $(P=0 \cdot 004)$, compared with following the control dairy products. No significant differences in iAUC for the TAG, glucose, NEFA or insulin responses were evident between diets. Chronic consumption of SFA-reduced, MUFA-enriched dairy products decreased the postprandial apoB response suggesting an impact of the FA-modified dairy on the metabolism of TAG-rich lipoproteins, which warrants further investigation.

This research was supported by the Medical Research Council (MR/K020218/1), with food in-kind from Arla Foods UK and AAK (UK) Ltd.

1. Markey et al. (2014) Nutr Bull 39, 161-171.

2. Bansal et al. (2007) JAMA 298, 309-16. 